\title{
Emergence of time in Loop Quantum Gravity
}

\author{
Suddhasattwa Brahma, ${ }^{1}$ \\ ${ }^{1}$ Center for Field Theory and Particle Physics, \\ Fudan University, 200433 Shanghai, China
}

\begin{abstract}
Loop quantum gravity has formalized a robust scheme in resolving classical singularities in a variety of symmetry-reduced models of gravity. In this essay, we demonstrate that the same quantum correction which is crucial for singularity resolution is also responsible for the phenomenon of signature change in these models, whereby one effectively transitions from a 'fuzzy' Euclidean space to a Lorentzian space-time in deep quantum regimes. As long as one uses a quantization scheme which respects covariance, holonomy corrections from loop quantum gravity generically leads to non-singular signature change, thereby giving an emergent notion of time in the theory. Robustness of this mechanism is established by comparison across large class of midisuperspace models and allowing for diverse quantization ambiguities. Conceptual and mathematical consequences of such an underlying quantum-deformed space-time are briefly discussed.
\end{abstract}

\section{Introduction}

It is not difficult to imagine a mind to which the sequence of things happens not in space but only in time like the sequence of notes in music. For such a mind such conception of reality is akin to the musical reality in which Pythagorean geometry can have no meaning.

— Tagore to Einstein, 1920.

We are yet to come up with a formal theory of quantum gravity which is mathematically consistent and allows us to draw phenomenological predictions from it. Yet, there are widespread beliefs among physicists working in fundamental theory regarding some aspects of such a theory, once realized. These premonitions about the final form of a quantum gravitational theory comes from two somewhat mutually exclusive ideas. Firstly, experience in dealing with other fundamental forces of nature and their quantization, which have resulted in the Standard Model of Particle Physics, has led to certain expectations regarding the outcome of quantizing gravity. This is, of course, quite natural and what one expects to happen. The other major source of prejudice, however, comes from the rather maverick nature of gravity as described through its classical theory - General Relativity (GR). The foundational idea of relativity that gravity is geometry requires a rather careful handling

\footnotetext{
*Submitted for "Space and time after quantum gravity" essay contest

$\dagger^{\dagger}$ e-mail address: suddhasattwa.brahma@gmail.com
} 
in the way one approaches to 'quantize' such a theory. It has often been said that GR is one of the most beautiful theory in physics; and like any beautiful object, its significance lies in the eyes of the beholder. Let us give an example to illustrate this point.

Particle physicists considered the primary difficulty of quantizing gravity to be the ultraviolet (UV) divergences which cannot be cancelled by a finite number of counterterms, as is done for every other fundamental force. This is what is commonly known as the non-renormalizability of gravity [1]. Thus, they went looking for a more suitable way to describe the dynamics of a massless, spin-2 particle - namely, the graviton - just as for other such gauge bosons in the Standard Model. Broadly speaking, this search has culminated in the foundation of String Theory [2, 3, 4] which describes not only gravity, but also all the other fundamental forces through the spectra of string oscillations, as this fundamental object (the string) travels through a $d$-dimensional spacetime 1 . Implementing the excitations as extended objects (strings) with nonlocal interactions, one gets rid of the infinities, the unwieldy mathematical objects standard field theory is beset with. Without going through the extremely rich history of the development of String Theory in detail, it is safe to say that one of the most ambitious goals for a String theorist is to unify all the fundamental forces of nature via one common framework. In the more recent past, String Theory has also led to rather amazing mathematical dualities whereby one is allowed to study the quantum gravity theory in a bulk space-time by simply examining a quantum field theory, without gravity, on its boundary [5]. This remarkable feature, termed the 'GaugeGravity duality', or more generally the 'Holographic Principle', can be looked upon as one of the most 'beautiful' artifacts of quantum gravity, arising from underlying mathematical symmetries.

On the other hand, relativist: $2^{2}$ take the idea of background independence as a guiding principle for quantizing gravity rather seriously [6]. The idea here is that one cannot quantize the graviton as a particle moving on a specified space-time, as is done for the other fundamental gauge fields in nature. Rather gravity supplies us with both the stage as well as the actors on it: the background space-time as well as the particle mediating gravity has to emerge from the fundamental theory [7]. This 'beautiful' idea led to several theories which do not assume a fixed form of a background space-time, most notably in Loop Quantum Gravity (LQG). Under the rather minimal requirement of a differential manifold, a suitable reformulation of GR is proposed such that the fields describing it do not, à priori, require a background metric. This does not imply that there is no background independence in String Theory; it is, however, implemented in a much more indirect manner and is in a rather nascent stage at the moment (for instance in String Field Theory [8]). It is important to emphasize that background-independence, as a motivation, is not a monopoly of LQG, but there are other approaches, such as Aymptotic Safety or Causal Dynamical Triangulations, which implement this feature in different ways. We mention this example to cite what was at the heart of looking for an alternative to String Theory.

\footnotetext{
${ }^{1}$ Historically, String Theory did come about to describe Strong Interactions before one found a more suitable candidate for the latter in Quantum Chromodynamics.

${ }^{2}$ Obviously, both these classifications of 'particle physicists' and 'relativists' are made rather loosely in order to make a larger point.
} 
Similarly, although direct manifestations of the 'Gauge-Gravity duality' is yet to be revealed in LQG, there is nothing to suggest that such a principle is lacking in the theory ${ }^{3}$ (see for instance, [9]).

So we find that two of the major approaches to quantum gravity not only do not share their technical and conceptual foundations but even diverge in their ambitions for the resulting theory. And, of course, the diversity in research for a quantum theory of gravity is also rather rich with many of the other approaches (not mentioned here) bringing distinct aspects of gravity into play. How can such ideas converge in their expectations from the final version of their individual quantum gravity theories? In order to understand this, let us first recall what quantizing the other fundamental forces have taught us. Quantum theories, typically, help us in resolving singularities which appear in their classical counterparts. This general expectation, as can be understood from its origin, is shared by all theories of quantum gravity. Since some well-defined physical quantity, such as energy density, diverges at the singular point, one concludes that some of the laws of physics have broken down at the singularity and we require a new set of rules to describe the dynamics in those regimes. Let us recourse to a (perhaps over-)simplified example to make our case. Hooke's law, describing the force exerted by a spring, can be written as $k=F / x$. This implies if we are to calculate the tension in the spring between two infinitesimally close points, then it would indeed be infinite! But this is not a true infinity but rather only a mathematical manifestation of the fact that we have applied the classical Hooke's law beyond its realm of validity. This can be seen if one recalls that the spring is built out of atoms, and once we consider subatomic lengths, laws of atomic physics has to come in to replace the classical Hooke's law. Thus, the fundamental minimum distance scale of atomic spacings (which is something that can be derived, and is not postulated, from the Schrödinger equation governing the quantum theory) comes in to save the day. A similar thing is expected to happen for the (curvature) singularities found in GR [10, mostly famously the initial singularity in cosmology termed the 'Big Bang' and the one found inside the core of black holes. Any quantum gravitational theory attempts to bring in a new set of laws which would cure the theory of such (classical) pathologies.

Next we have to deal with the more subtle point of the expectation of a non-classical, 'fuzzy' nature of quantum space-times, which is also expected by and large from almost all theories of quantum gravity This is a more surprising common factor between quantum gravity theories since it is realized through distinct mathematical procedures having origin in the different theories. Almost all background independent quantizations of gravity talk of a discrete, granular description of space-time at some fundamental level. The guiding principle for these theories is that space-time, on the most primordial level, is an irregular substratum made up of the fundamental building blocks, or the atoms of space-time. Naturally, the explicit form of these elementary degrees of freedom depend on the specific framework one deals with ${ }^{5}$ but, on the whole, one loses the smooth, continuous description

\footnotetext{
${ }^{3}$ However, key difference between the two theories remain that LQG is less ambitious than String Theory in not attempting an unification of all the known forces in nature.

${ }^{4}$ One notable exception is the Asymptotic Safety program [11.

${ }^{5}$ In LQG, for instance, one has discrete spin-network states giving rise to a discrete area and volume
} 
of classical backgrounds geometries on sub-Planckian scales. On the other hand, although String Theory is at least formulated over smooth manifolds, there is a growing consensus that space-time in this case (and may indeed be for any consistent theory of gravity) is built out of quantum entanglement (for instance, see [12]). Even proposals that String Theory is an coarse-grained approximation of a more statistical phenomenon of microscopic degrees of freedom, with gravity being an emergent interaction, has been gaining ground [13]. The bottom line is that all of this points towards a description of gravity on fundamental scales which is quite exotic compared to its classical counterpart as a smooth space-time continuum.

In this essay, we aim to use the input of singularity resolution from LQG and show that, demanding mathematical consistency leads to the emergence of non-Riemannian geometry in the theory. Specifically, our goal is to show that 'time' is an emergent concept in LQG, in the sense that one effectively transitions from a four-dimensional 'fuzzy' Euclidean space to the usual $(3+1)$-dimensional Lorentzian space-time in high curvature regimes. Although this shall be demonstrated only for symmetry-reduced systems in LQG, care needs to be taken that they are not oversimplified toy models, bland enough to miss key subtleties of the full theory. We emphasis that our goal is not to comment on the robustness of singularity resolution in LQG, which has been established to a great extent in the existing literature (for instance, see [14, 15, 16, 17]), but rather to only extract a crucial ingredient commonly required for tackling singularities in the theory. The other input to be assumed is that the theory avoids quantum gravitational anomalies and satisfies a well-defined notion of covariance (adapted to a canonical setup) which is a strict requirement, not just of LQG, but any consistent quantum gravity theory ${ }^{6}$. These two minimal requirements, when combined, would be shown to give rise to a rather remarkable change in our understanding of the underlying space-time, leading to emergence of time in LQG.

\section{Singularity Resolution in LQG}

Setting aside the mathematical beauty of GR, it has also been an extremely successful theory and has passed all proposed experimental tests with flying colors thus far. Yet, a more fundamental theory is required which incorporates not only the dynamical nature of geometry but also the features of quantum physics. Indeed, a brief glance at Einstein's equations, $G_{\mu \nu}=(8 \pi G / 3) T_{\mu \nu}$, is enough to convince one of that. Since the right-hand side of the equation consists of 'quantum' matter described by the Standard Model, the left-hand side must be replaced by some suitable 'quantum' version of gravity. The more obvious reason for invoking a quantum gravity theory obviously arises when dealing with curvature singularities contained in solution to Einstein's equations, where the equations themselves fail. This is a more serious problem than the divergences which arise in other gauge theories describing, for instance, the electromagnetic field. In Minkowskian physics,

spectra [6].

${ }^{6}$ What we mean precisely by this shall be spelled out more explicitly in a later section but it is important to note that this is different from the idea of Lorentz covariance for a flat, Minkowski space-time. 
if such a field becomes singular at a given point, it does not affect the underlying spacetime and, consequently, has no effect on the evolution of the other fields in the theory. However, since gravity is geometry according to GR, when the gravitational field becomes singular, the picture of the continuum space-time itself breaks down and all of physics gets disrupted. Now, we do not have a stage any longer on which to describe the dynamics of the other matter or gauge fields. This points towards the fact that our picture of gravity as a smooth continuum of space-time must itself not be applicable to points arbitrarily close to such a singularity and what we require is a quantum theory of geometry (see, for instance, [6, 18] for details).

Let us focus on the simple example of the $k=0$, FLRW geometry to understand how singularity-resolution takes place in LQG [19, 20. (In this context, we would refer to this theory as Loop Quantum Cosmology (LQC) from now on since it is indeed the quantization of a symmetric sector of GR inspired by techniques from LQG rather than a 'coarse-graining' of the cosmological degrees of freedom from the full quantum theory.) The requirements of consistency from LQC are twofold: On one hand, the quantum theory needs to have a regular behaviour for the quantum state in the higher curvature regime and resolve the Big Bang singularity. On the other hand, these quantum corrections, which are strong enough to overcome the gravitational collapse due to Planck scale energy densities, have to die off extremely fast beyond the Planck scale so that we recover the well known behaviour of GR. Both these conditions are met by LQC and thus we have a welldefined quantum cosmological model. Inside the deep quantum regime, the density and curvature reach a maximum value, as opposed to increasing indefinitely as in the classical theory due to novel quantum geometry effects. This might be interpreted as an 'effective' repulsive force which overcomes the classical gravitational attraction and thus resolves the singularity by preventing the quantum evolution from going to the singular point. In the full quantum theory, one can show that singularity-resolution might be seen as the zero volume state getting decoupled from the dynamics of the quantum state corresponding to the universe. Although the main achievement of LQC is to incorporate novel quantum geometry corrections, heuristically adapted from LQG in the form of a minimum non-zero value of area (or the area-gap) in the left-hand side of the Einstein's equation, we can shift the correction to the right-hand side to facilitate comparison with the standard Friedmann equation, which now reads

$$
H^{2}=\frac{8 \pi G}{3} \rho\left(1-\frac{\rho}{\rho_{c}}\right),
$$

where $H$ is the Hubble parameter and $\rho$ is the energy density, which gets an upper bound (the critical density, $\rho_{c}$, which is proportional to $M_{P l}^{2}$ ) from these quantum effects.

Although we describe the broad picture of singularity-resolution in the specific case of LQC above, the mechanism remains the same for other models where it has been possible to do the same. Specifically, singularity resolution has been achieved for a variety of cosmological models [21, 19] such as the Bianchi models, FLRW geometries with cosmological constants, to Gowdy systems with additional symmetries 22] and for open or closed universes, in addition to the flat case mentioned above. Recently, similar techniques have been 
applied to black hole solutions, such as Schwarzschild [17] or CGHS models [23], with the remarkable success of resolving the singularity inside the cores of such black holes much like in the same manner as described above. However, for our purposes, we do not need to go into the full quantum theory of any of these cases in detail. Instead, we focus on the most crucial ingredient in LQG which leads to such singularity resolution across a variety of different models.

In LQG, we have well-defined operators for holonomies (or parallel transports of connections) on the kinematical Hilbert space but not for the connections themselve: ${ }^{7}$. Thus one requires a different way of expressing connections on the Hilbert space via their holonomies. Curvature of the connection, which shows up in the Hamiltonian of the theory, have to be regularized in terms of these holonomies. Indeed, a direct consequence of this can be seen in the minisuperspace quantization of LQC. The Wheeler-de Witt (WDW) theory in this case, which arises from the Schrödinger quantization of the quantum mechanical phase space, is known to suffer from the same pathologies as the classical solution as far as the big bang is concerned. On the other hand, the Stone von-Neumann uniqueness theorem guarantees that the quantum kinematics for a finite dimensional phase space isomorphic to $\mathbb{R}^{n}$ (such as the quantum cosmological model) is unique. The way LQC can go past this obstruction is precisely via violating the assumptions of weak-continuity from the von-Neumann theorem and thus, essentially, giving rise to a new form of quantum mechanics. In effect, all of these mathematical rigor can be pinned down to give one effect: the inclusion of holonomy corrections in the theory.

In an 'effective' theory of a loop-quantized model, the idea is to replace the connection (or, in practice, extrinsic curvature components) by a polymerization function in the Hamiltonian of the theory [24]. This polymerization function is obviously not chosen ad $h o c$, but rather through a rigorous regularization of the curvature components in a specified representation of the internal gauge group (SU(2)), along with inputs of the area-gap from the full theory. Typically the holonomies are calculated in the fundamental spin-(1/2) representation of $\mathrm{SU}(2)$ and the polymerization function, for some extrinsic curvature component $K$, takes the form

$$
K \rightarrow \frac{\sin (\delta K)}{\delta},
$$

where $\delta$ is related to the Planck length in a specified manner. This kind of non-perturbative correction, coming from LQG, is commonly known as holonomy correction (or equivalently, modification) function. Although the sine function has been obtained explicitly in the case of LQC, for the spin-(1/2) representation, we can allow for more general quantization ambiguities and replace connection components by any local and bounded function of it. This type of correction plays the most crucial role in singularity resolution and the bounded nature of the function comes from this requirement. It is important to emphasize that LQG does not resolve singularities by incorporating some arbitrary bounded functions of the connection, but rather derives them in some symmetry-reduced models. For the purposes

\footnotetext{
${ }^{7}$ Mathematically, this implies that the holonomy operators are not weakly continuous.
} 
of this essay, we simply choose to not work with a specific form of this function but rather keep it arbitrary, thus demonstrating that our conclusions are not tied to some specific fine-tunings arising from a particular model in LQG but is rather general in its ambit. Therefore, the first input for us is going to be incorporating such holonomy correction functions which lead to singularity resolution (and is thus bounded) $K \rightarrow f(K)$.

Before concluding this section, we need to make a comment about why this function has to be chosen to be local. It is tied to the same reason that we are yet to have a consistent formulation of any canonical quantum gravity theory in $(3+1)$-dimensions. It is only known how one can loop-quantize simpler, symmetry-reduced models and, only in such cases, can singularity resolution be demonstrated manifestly. In LQG, one is yet to understand how to explicitly evaluate holonomy operators in inhomogeneous directions, along edges of spin-network states. Although the full technical details of this problem is far beyond the scope of this essay, we shall revisit this issue in the simplified arena of Schwarzschild space-times.

\section{Covariance in canonical quantum gravity}

It is well-known that coordinate freedom is one of the stepping stones of GR (and, indeed, of all general covariant theories going beyond GR), i.e. the theory remains invariant under local diffeomorphisms $x^{\mu} \rightarrow x^{\mu}+\xi^{\mu}(x)$. LQG is based on a Hamiltonian formulation of gravity, where space-time is foliated into spatial hypersurfaces evolving along a time parameter. However, in canonical theories, coordinate transformations of space-time tensors are replaced by gauge transformations which generate deformations of the spatial hypersurfaces in full space-time. The Hamiltonian (or scalar) constraint is defined as the one which generates deformations along the direction normal to the hypersurface while the (spatial) diffeomorphism constraint generates those along tangential directions. In generally covariant theories, these four smeared constraints (per point) satisfy the (Dirac) hypersurface deformation algebra 8 , These constraints satisfy a 'first class' system forming a closed algebra, which implies that the Poisson brackets of the constraints vanish on the constraint surface (see [18] for details).

The phase space of canonical gravity is formed by the metric on the spatial hypersurface $q_{a b}$ and its conjugate momenta proportional to the extrinsic curvature components $K_{a b}$. The full space-time metric is typically parametrized by a lapse function $M$, the shift vector fields $N^{a}$ and the spatial metric $q_{a b}$. The arbitrary function $M$ labels the Hamiltonian constraint while $N^{a}$ smears out the diffeomorphism constraint. The crucial observation is that the symmetry deforming the spatial hypersurfaces, tangentially along $N^{a}$ and along the vector $M n^{\mu}$ ( $n^{\mu}$ being the unit normal to the hypersurface), are equivalent to Lie derivatives along space-time vector fields therefore representing coordinate freedom. Gauge-covariance under hypersurface deformations (equivalent to the underlying

\footnotetext{
${ }^{8}$ Technically, it forms a Lie algebroid and not an algebra, a fact which we shall utilize in the next section. However, in the meantime, we shall keep referring to it as an algebra as is common in the physics literature.
} 
space-time diffeomorphisms) is ensured by the following algebra

$$
\begin{aligned}
\left\{D\left[N_{1}^{a}\right], D\left[N_{2}^{a}\right]\right\} & =D\left[\mathcal{L}_{N_{1}} N_{2}^{a}\right] \\
\left\{H[M], D\left[N^{a}\right]\right\} & =-H\left[\mathcal{L}_{N} M\right] \\
\left\{H\left[M_{1}\right], H\left[M_{2}\right]\right\} & =D\left[q^{a b}\left(M_{1} \nabla_{b} M_{2}-M_{2} \nabla_{b} M_{1}\right)\right] .
\end{aligned}
$$

The Eqs. (3, 4) demonstrate the action of infinitesimal spatial diffeomorphisms with the right-hand side given by the Lie derivatives $\mathcal{L}_{N_{1}} N_{2}^{a}=\left[N_{1}, N_{2}\right]^{a}$ and $\mathcal{L}_{N} M=N^{a} \partial_{a} M$. The last Eq. (5) complicates the intuitive geometric picturization due to the appearance of structure functions, in the form of the inverse of the spatial metric. The Hamiltonian constraint does indeed provide the time reparametrizations required to supplement the spatial diffeomorphisms generated by $D\left[N^{a}\right]$ to form the full space-time diffeomorphism symmetry of gravity, but only on the constraint surface.

It is important to emphasize that covariance, in the canonical context, is an off-shell property. Fields which do not satisfy the constraint equations $D\left[N^{a}\right]=0$ and $H[M]=$ 0 , as derived from GR, still require to be well-behaved so as to not violate the above algebra. This is in keeping with our understanding of four-dimensional symmetries from Lagrangian theories where to write down a covariant action one only needs to define a Lorentz-invariant measure and contract indices properly with any metric, without paying attention to whether they are solutions to Einstein's equations. The specific nature of the algebra of the constraints has to be satisfied by the constraint functions on the whole phase space and not just the constraint surface. Its form dictates the kinds of gauge transformations the constraints generate and how they are related to space-time properties. All of this is, however, well-understood in the classical formulation of GR as a canonical theory. An open question, recently addressed, is regarding the fate of such symmetries once quantum modifications from a particular theory are taken into account.

We first postulate the requirements for a canonical quantum theory of gravity to be covariant [25, 26]:

1. The classical Hamiltonian and diffeomorphism constraint, on including quantum corrections, must still act as generators which form a closed algebra free of quantum anomalies.

2. The quantum algebra of the new generators must have a classical limit which is identical to the classical hypersurface deformation one as defined in Eqs. (3) -5).

The first condition simply ensures that we do not violate the gauge symmetries of gravity in the quantum theory. If the quantum constraint operators (or equivalently, effective versions of them including quantum corrections) still form a first-class system then we have the same number of gauge conditions required to eliminate spurious degrees of freedom as in the classical case. Thus the gauge generators, in the quantum theory, would also lead to the same dimension of the solution space and thereby avoids gauge anomalies. Since the absence of anomalies is the requirement for any consistent quantum (gauge) theory, condition (1) is the corresponding one for a quantum version of gravity viewed as a gauge 
theory. The second condition ensures that what we end up with, the quantum gravity theory, is a consistent quantum theory of space-time. This is an even stricter requirement since this ensures that we end up with a theory which gives the correct space-time structure in the classical regime. While the first condition deals alone with the important issue of anomaly-freedom in quantum gravity theories, both of them together ensure that one ends up with a covariant quantization of gravity. It is conventional to assume that once one can get the quantum corrected brackets to have a closed algebra, then the quantization is covariant. What has been recently demonstrated is that a background-independent quantization requires not only anomaly-freedom, but also a well-defined classical limit so that the quantum theory is indeed one of space-time. This is indeed the difference of full quantum gravity theory from a theory of matter on a quantum-modified space-time. The latter by itself has severe requirements that the gravitational and the matter forms closed first-class systems whereas the former not only requires this, but also that they have a matching version of covariance. Naturally, this has severe ramifications for the way one can covariantly couple matter to such quantum theories, which is beyond the scope of this essay. As a final reminder, we stress that our key requirement is not that the theory remains Lorentz invariant, which turns out to be a deformed symmetry in this context [27], but simply that the gravitational gauge conditions are not violated.

We end this section with an important caveat: One cannot see the effects of covariance in a minisuperspace model. One deals with homogeneous degrees of freedom alone in such systems and thus the diffeomorphism constraint is trivially satisfied for such models. Covariance cannot be addressed in such models because, owing to lack of inhomogeneities, the relationship between both temporal and spatial variations of fields are absent in them. Mathematically, since the diffeomorphism constraint is trivially zero, one cannot examine any behaviour of the constraint algebra in such settings. As a consequence, no restrictions on the dynamical equations of wavefunctions can be imposed by demanding covariance in such models and any putative quantum effect can be included at will. However, such arbitrary quantum modifications would result in a minisuperspace model that cannot be embedded within a larger covariant full theory. Thus, we shall have to deal with models which have at least one inhomogeneous component. In fact, all known models of LQG which resolve classical singularities (and are not completely homogeneous) are of this form. Thus, we shall hereafter restrict ourselves to such midisuperspace models.

\section{Emergence of Time in LQG}

We have all our ingredients and are ready to state our main result. From Sec (2), we infer that singularity resolution in LQG typically introduces a bounded function for extrinsic curvature components. We keep our assumptions to a minimum and do not even fix a specific form for the holonomy correction function. This kind of a general ansatz, requiring

only that the correction function is bounded, then suggests that we are gathering the least information necessary from the particular theory (LQG) and keeping ample room for improvements to the quantization scheme within it. Our second requirement, following Sec 
(3) is the necessary condition that the quantization procedure is space-time covariant, in a well-defined sense. Based on these two rather general assumptions, we shall show that there is a remarkable deformation to the underlying space-time structure for such quantum modifications.

\subsection{Explicit example: Spherically symmetric gravity}

It is easiest to first concentrate on a concrete example to illustrate our point and then state the general result for other such systems. For spherically symmetric gravity adapted to Ashtekar-Barbero variables [28], one has a two dimensional phase space spanned by the triad variables $E^{x}$ and $E^{\phi}$ in the radial and angular directions respectively, along with their canonically conjugate extrinsic curvature components $K_{x}$ and $K_{\phi}$. There is only one nontrivial component of the diffeomorphism constraint, given by

$$
D\left[N^{x}\right]=\frac{1}{G} \int \mathrm{d} x N^{x}(x)\left(-\frac{1}{2}\left(E^{x}\right)^{\prime} K_{x}+K_{\phi}^{\prime} E^{\phi}\right),
$$

while the gravitational spherically symmetric Hamiltonian constraint takes the form

$H[M]=-\frac{1}{2 G} \int \mathrm{d} x M(x)\left(\left|E^{x}\right|^{-\frac{1}{2}} E^{\phi} K_{\phi}^{2}+2\left|E^{x}\right|^{\frac{1}{2}} K_{\phi} K_{x}+\left|E^{x}\right|^{-\frac{1}{2}}\left(1-\Gamma_{\phi}^{2}\right) E^{\phi}+2 \Gamma_{\phi}^{\prime}\left|E^{x}\right|^{\frac{1}{2}}\right)$,

with the spin-connection given by $\Gamma_{\phi}=-\left(E^{x}\right)^{\prime} / 2 E^{\phi}$. The prime denotes a derivative with respect to the radial coordinate. We have suppressed the dependence of the field variables on the radial coordinate in the above expressions (due to spherical symmetry, we can safely integrate out their dependence on the angular coordinates). Thus we have a system of 2dimensional phase space with 2 first-class constraints, thus resulting in no local physical degrees of freedom (the only global degree of freedom is the ADM mass of the system). The usual spatial metric of the system can be written in terms of the Ashtekar-Barbero variables as

$$
\mathrm{d} q^{2}=\frac{\left(E^{\phi}\right)^{2}}{\left|E^{x}\right|} \mathrm{d} x^{2}+\left|E^{x}\right|\left(\mathrm{d} \theta^{2}+\sin ^{2} \theta \mathrm{d} \varphi^{2}\right) .
$$

The classical constraint algebra can be easily evaluated using the Poisson structure of the phase space

$$
\left\{K_{x}(x), E^{x}(y)\right\}=2 G \delta(x, y), \quad\left\{K_{\phi}(x), E^{\phi}(y)\right\}=G \delta(x, y) .
$$

The only quantity of the inverse spatial metric which shows up as a structure function in the algebra is $q^{x x}=\left|E^{x}\right| /\left(E^{\phi}\right)^{2}$ since the only derivative that gives a non-zero result is along the radial coordinate.

We now proceed with our program of replacing the (angular) extrinsic curvature components with local functions of itself, i.e. $K_{\phi} \rightarrow f\left(K_{\phi}\right)$ in the Hamiltonian constraint. As mentioned before, we do not consider holonomies corresponding to the $K_{x}$ variables since 
they are calculated along the edges of a (one-dimensional) spin-network and are, therefore, non-local in nature and difficult to implement [29]. However, it has been shown that one can suitably reformulate the constraints to eliminate the $K_{x}$ variable from the Hamiltonian constraint altogether and only have the $K_{\phi}$ one [17]. Thus, we can consider the point-wise holonomy operators corresponding to the $K_{\phi}$ component alone, which act locally at the nodes of the spin-networks, without loss of generality. With these modifications, the effective Hamiltonian constraint takes the form [26, 30]

$$
\begin{aligned}
H[N]= & -\frac{1}{2 G} \int \mathrm{d} x N(x)\left(\left|E^{x}\right|^{-\frac{1}{2}} E^{\varphi} f_{1}\left(K_{\varphi}\right)+2\left|E^{x}\right|^{\frac{1}{2}} f_{2}\left(K_{\varphi}\right) K_{x}\right. \\
& \left.+\left|E^{x}\right|^{-\frac{1}{2}}\left(1-\Gamma_{\varphi}^{2}\right) E^{\varphi}+2 \Gamma_{\varphi}^{\prime}\left|E^{x}\right|^{\frac{1}{2}}\right) .
\end{aligned}
$$

Note that we do not replace both the instances where $K_{\phi}$ shows up in Eq. (7) with the same function but allow for even more generalities by plugging in different correction functions $f_{1}$ and $f_{2}$ (the classical expressions are recovered for $f_{1}\left(K_{\phi}\right)=K_{\phi}^{2}$ and $f_{2}\left(K_{\phi}\right)=K_{\phi}$ ). However, we keep our diffeomorphism constraint unmodified from the classical case. The reason for this is that, in LQG, one does not have an infinitesimal quantum operator generating spatial diffeomorphisms represented on spin-network states but rather spatial diffeomorphism invariance is implemented in the full quantum theory through finite unitary transformations (See [31] and references therein for an attempt to define an infinitesimal diffeomorphism constraint in LQG). The Hamiltonian constraint, on the other hand, acts infinitesimally only on diff-invariant spin-network states in LQG. We are interested in questions regarding the covariance of the theory and thus are interested in the off-shell structure of the constraint algebra, which should be a largely representation-independent question and not depend specifically on the spin-network states, the latter being after all only a choice of basis. In other words, we assume that the flow of the diffeomorphism constraint is not crucially different from the classical one as is done in all models of LQG which achieve singularity-resolution?

We are now ready to calculate the brackets between the quantum-corrected constraints to find the resulting form of the constraint algebra. The algebra of basic variables is now modified due to the inclusion of holonomy corrections for the angular connection component

$$
\left\{f\left(K_{\phi}\right)(x), E^{\phi}(y)\right\}=G\left(\frac{\mathrm{d} f}{\mathrm{~d} K_{\phi}}(x)\right) \delta(x, y) .
$$

There is à priori no reason to assume that the quantum-corrected constraints would even form a closed algebra and thereby satisfy the requirement of anomaly-freedom. However, it turns out that the calculation of the brackets reveals two things. Firstly, we find a condition relating the two arbitrary holonomy modification functions and hence they are not both independent. This condition is a requirement for anomaly-freedom, which gives

\footnotetext{
${ }^{9}$ This presupposes that three-dimensional space, but not necessarily space-time, retains some features of the classical structure.
} 
[26, 30]

$$
f_{2}\left(K_{\phi}\right)=\frac{1}{2} \frac{\mathrm{d} f_{1}}{\mathrm{~d} K_{\phi}} .
$$

More importantly, we find that the full algebra of the quantum-corrected constraints stays the same except for the bracket between two Hamiltonian constraints, which takes the form [26, 30, 32]

$$
\left\{H\left[M_{1}\right], H\left[M_{2}\right]\right\}=D\left[\beta q^{x x}\left(M_{1} \partial_{x} M_{2}-M_{2} \partial_{x} M_{1}\right)\right],
$$

where the classical structure function, $q^{x x}$, is modified by the factor

$$
\beta=\frac{1}{2} \frac{\mathrm{d}^{2} f_{1}}{\mathrm{~d} K_{\phi}^{2}}
$$

We make the following observations regarding this deformation of the quantum constraint algebra.

1. Since we kept both the holonomy modification functions arbitrary, there was no guarantee that the algebra would close. However, we find that it is not only anomalyfree but closure also requires that one of the function be expressed in terms of the other.

2. When we take the classical limit of the holonomy correction function $f_{1}$, we see that $\beta$ goes to 1 reproducing the familiar hypersurface deformation algebra. This remarkable result satisfies our condition for a covariant quantization.

3. Classically, the curvature component can increase infinitely, which in turn implies that the energy density gets infinite at the classical singularity. However, due to the inclusion of the holonomy modification from LQG, the function $f_{1}$ reaches a maximum and we avoid the classical singularity. The deformation function $\beta$ necessarily turns negative when the holonomy modification function precisely reaches its maximum. This is because $\beta$ is the second order derivative of a function which reaches its maxima. This simple, yet striking, feature of the deformation function has longranging ramifications.

Once the deformation function changes sign, the bracket between two normal deformations ${ }^{10}$ in the modified constraint algebra has the same sign as one gets from Euclidean gravity. This is referred to as signature change in the literature since, although we start from the usual assumptions of a Lorentzian space-time, due to quantum corrections from LQG, we end up in an Euclidean four-space in the deep quantum regime. This implies radical new ideas regarding the nature of underlying quantum space-time and a new perspective of its geometrical structure above Planck scales. At this point, it is sufficient

\footnotetext{
${ }^{10}$ Throughout this essay, we have been using the word 'deformation' to mean two different things, which should be clear from the context in which it is used.
} 
to point out that we have successfully derived a deformed notion of covariance for the quantized system, whereby one modifies gauge transformations by quantum corrections but does not allow them to be violated. As promised, we only introduced the crucial feature of bounded holonomy operators from LQG to achieve this goal. As a consequence of this, 'time' appears as an emergent parameter below some energy scale during our effective transition from an Euclidean to the Lorentzian phase. Although all the calculations shown above is for effective constraints for simplicity, i.e. we do not take explicit quantum operators corresponding to the constraints into consideration, the same result holds when operator effects are taken into consideration as shown in [30]. Thus this result is certainly not the manifestation of some semiclassical approximation introduced within this scheme. (It has also been shown that fluctuations and higher moments of the quantum state cannot introduce perturbative loop corrections which can deform the structure functions of the constraint algebra [33].)

\subsection{Ubiquity of signature change in LQG}

Since we have shown that time emerges at a some particular scale for spherically symmetric gravity when effects required for singularity resolution are taken into account, how can one be sure that this is not due to some additional quantization choices introduced by us? We can answer this question in two ways. Firstly, the only assumption used by us, as shown in the previous subsection, was that of bounded curvatures which is at the heart of singularity resolution in LQG. However, although our framework was kept extremely general on purpose, we can do even better to exhibit the robustness of our claims. The same spherically symmetric system, or equivalently the Schwarzschild black hole model, has been quantized based on a completely different, but classically equivalent, set of firstclass constraints where the most complicated part of the constraint algebra had been Abelianized [17]. In other words instead of working with the familiar $H[M]$ and $D\left[N^{x}\right]$ constraints as introduced in Eqs. (7, 6), the quantization was performed over a newly defined $C[L]$ constraint and the usual diffeomorphism $D\left[N^{x}\right]$ constraint. For this set, firstclass algebra has the same form for Eqs. (3, 4) (with $H$ replaced by $C$ ) while the last relation is replaced by $\left\{C\left[L_{1}\right], C\left[L_{2}\right]\right\}=0$. Thus we do not have the structure functions appearing anymore and we have a true Lie algebra. (This is possible by choosing some judicious linear combination of the old constraints to define the new constraint $C[L]$.) It has been further shown that the Schwarzschild singularity has been resolved for a quantum theory based on these constraints, after polymerizing them according to LQG. One would then imagine that signature change would be impossible for such a system due to the disappearance of the structure functions. However, as explained in [26], requiring that the resulting quantum theory be covariant (as defined in Sec (3p), one can show that not only does the structure functions reappear and get deformed but also we have signature change for this model. Additionally, it was shown that if the holonomy correction functions were kept arbitrary for this partially Abelianized system, then the restriction (12) is the same one that is obtained even for the new system. Since the technical details of the two systems were largely different except for the choice of bounded functions for curvature components, 
we conclude that signature change (and consequently, the appearance of time) does not depend on any additional regularization choices made by us in the previous subsection but is a rather general result for the Schwarzschild black hole model in LQG.

However, it might still be that there is some magical coincidence that leads to the fortuitous notion of deformed covariance in the spherically symmetric space-time and therefore signature change is nothing but a manifestation of the symmetry of the system. To verify that this is not the case, we can go back to the original cosmological setup where singularity was first resolved within LQG. However, as explained before, one cannot ask questions regarding covariance in homogeneous models, the traditional setup for LQC. Fortunately, nature also demands that our universe is not exactly homogeneous but rather has small inhomogeneities which leads to formation of galaxies. We should then loop quantize a system which has perturbative inhomogeneities on top of a homogeneous FLRW background in cosmology to get a more realistic picture. Indeed such a quantum scheme leads to resolution of singularties once again through a non-singular signature change (see, for instance, [34, 35]). The fundamental setup of these models is quite different from the one considered above in that one only introduces holonomy correction functions for the background connection component but leaves classical expression for the perturbative inhomogeneous extrinsic curvatures. Yet, one finds that a bounded version of background curvature component to be a sufficient condition for signature change in these models.

Finally, there is a vast arena of models in LQG which have recently been examined to show that singularity resolution cannot be divorced from signature change for real valued connections [36, 37, 27]. In particular, a class of models shown to manifest signature change would be all 2-dimensional dilaton models which includes the Callan-GiddingsHarvey-Strominger (CGHS) black hole solution. Another example would be the (polarized) Gowdy system with local rotational symmetry. We mention these models in particular since the classical singularity in these models have been shown to be satisfactorily resolved through loop quantization [23, 22. It has been demonstrated that for all these models the classical singularity is, in fact, replaced by a non-singular signature change and thus time appears in all these models below some energy scale. Indeed, this work has been generalized even further to a general midisuperspace model, with one direction of inhomogeneity and no local physical degrees of freedom, to show that even for such a general model (which includes all the above examples and even more class of models with not well-defined classical analogs), holonomy corrections from LQG results in deformation of the structure function in a way that leads to signature change. The robustness of these findings has also been checked against different choices of canonical variables and for different choices of arbitrary dilatonic potential terms. It appears that time is indeed an emergent parameter, at least within the class of models for which one can have singularity resolution in LQG.

\section{Discussion}

Having shown that the same modification that resolves singularity in LQG is also responsible for signature change, at least in all models in which classical singularities can be 
resolved in LQG, let us discuss some of the aspects of our findings.

\subsection{Mathematical basis for emergence of time}

Our essay shows novel features in the background space-time structure in quantum regimes due to the modification of the classical structure functions appearing in the quantumcorrected constraint algebra. However, it is natural to assume at first glance that one can absorb the deformation factor $\beta$ in the inverse of the spatial metric to get an 'effective' spatial metric $\tilde{q}^{a b}:=\beta q^{a b}$. However, such an effective metric from the relation

$$
\left\{H\left[M_{1}\right], H\left[M_{2}\right]\right\}=D\left[\tilde{q}^{a b}\left(M_{1} \nabla_{b} M_{2}-M_{2} \nabla_{b} M_{1}\right)\right],
$$

cannot be part of a space-time metric to form a classical line element of the form $\mathrm{d} s^{2}=$ $-N^{2} \mathrm{~d} t^{2}+\tilde{q}_{a b}\left(\mathrm{~d} x^{a}+N^{a} \mathrm{~d} t\right)\left(\mathrm{d} x^{b}+N^{b} \mathrm{~d} t\right)$. It is because the modified gauge transformations generated by the quantum corrected constraints for $\tilde{q}_{a b}$ do not match the coordinate transformations of the infinitesimal $\mathrm{d} x^{a}$. This complication notwithstanding, one might venture to find field redefinitions of the the lapse, shift, the original spatial metric and the extrinsic curvature, or a combination thereof, to absorb $\beta$ and recover the classical hypersurface deformation algebra. In other words, one can try to define new field configurations as combinations of the old ones which would then give rise to the same constraint algebra structure as the classical case. Even if one is successful in finding such transformations, it does not imply that the deformation we find is spurious: It would still affect the equations of motion of particles on these deformed space-times. However, it would imply that the background can be treated effectively as a Riemannian geometry. This does not mean that the background space-time does not pick up quantum corrections, but rather that in spite of such modifications they retain some notion of classical space-time structures in that they can still be expressed as Riemannian manifolds. However, it is notoriously difficult to come up with different canonical transformations to check whether $\beta$ can be absorbed by one of them and therefore still retain a Riemannian geometry. (Even if one cannot find one such transformation for a given system, it does not rule out the possibility of one existing since there are infinite number of transformations which can be applied to define new variables from the old ones.)

Recently, this issue was addressed using a different approach [38]: using the well formulated theory of Lie algebroids since the hypersurface deformation brackets provide an example of one. Indeed, irrespective of the specific choice of the quantum gravity theory, it was possible to classify different inequivalent space-time structures which cannot be related by algebroid-morphisms. Since we do not want to obfuscate our central findings with more mathematical structures than necessary, we do not reproduce the details of that proof in this essay. However, we shall nevertheless state our main result, as follows. Although it would seem that an arbitrary phase-space deformation function $\beta$ would imply virtually unrestricted quantum corrections, however, only $\operatorname{sgn}(\beta)$ remains the unique choice after equivalence class of algebroids are taken into account. This has two main implications. Firstly, as long as one has a deformation function that does not change sign, it is possible, 
in principle, to absorb this factor using some transformation and thus has an effective Riemannian structure. However, once $\beta$ changes sign, then one cannot absorb it globally and a new version of quantum space-time is obtained. In such a case, one has distinct Lorentzian and Euclidean patches, which form non-isomorphic Lie algebroids. This is the mathematical reason behind the emergence of time in such theories. Keep in mind, that this entire mathematical analysis has been done without reference to any specific quantum gravity theory. Our only input from LQG is to provide a quantum modification function which results in a sign changing $\beta$, which triggers signature change.

Let us finally make some speculative comments regarding the emergence of time in these models of LQG. As has been throughout emphasized, for every symmetric model of LQG where one can resolve the classical singularity, one can explicitly prove signaturechange. These are precisely systems which model real-world cosmology (such as FLRW geometry with small inhomogeneities) or black holes (such as Schwarzschild or CGHS). Let us focus on the cosmological example first. Since the space-time now changes sign, a natural question to ask is how does the cosmological perturbations propagate on top of such a quantum space-time. Firstly, in a strict sense, perturbations cannot 'propagate' in the Euclidean phase due to a lack of a sense of time in this phase. However, one might identify one of the spatial directions as the one which undergoes this 'physical' Wick rotation to emerge as a time parameter. In that case, the change (we purposefully avoid using the word 'evolution') of the perturbations may still be calculated with respect to this parameter. However, a better method to think about the cosmological setting may be the following. For actual physical predictions, it might be sufficient to specify some initial value for cosmological perturbations infinitesimally close to the signature-changing hypersurface on the Lorentzian side and then evolve them on to the beginning of the inflationary phase. This way the initial state for inflation can be specified accurately from the underlying quantum gravity theory since there is no conceptual problem in evolving them throughout in the Lorentzian phase. Furthermore, to obtain some intuitive understanding of the deep quantum regime, one may even evolve them 'backwards' to evaluate their value on the signature-changing hypersurface. This would then be matched onto the values of the these perturbation modes on the boundary of the 'fuzzy' Euclidean phase.

The overall picture with black holes is somewhat less clear at present. However, if the core of black holes display a Euclidean region, it implies that there is no deterministic evolution through this high curvature region. While singularity in the form of diverging curvature may be avoided, a challenging question persists in the sense of a space-time incompletely determined by initial data. In order to extend space-time across the Euclidean region, one requires additional data on the part of its boundary that borders on the future Lorentzian space-time. This additional requirement is reminiscent of other proposals of black-hole models, for instance stretched horizons', introduced in the context of the black hole complementarity principle. In any case, the detailed analysis of anomaly-free black holes within LQG points towards a much subtler non-singular description of quantum space-time than usually postulated in simplified bounce models. Just as an outside observer finds the stretched horizon as a membrane, first storing and later releasing information in the form of microphysical degrees of freedom, additional information is encountered once 
an observer moves into the future of a Euclidean region embedded in space-time. However, it should be pointed out that in the case of black-hole models of LQG, there is as yet no microscopic theory that would restrict or determine possible data around Euclidean regions.

\subsection{Necessity of deformed covariance}

In their seminal work, Hojman, Kukař and Teitelboim [39, 40] had shown that starting from the classical hypersurface deformation algebra, one can uniquely get the Einstein Hilbert action (up to the cosmological constant), if one restricts to second order derivatives of the field variables. This remarkable result shows the uniqueness of GR as a covariant gravitational theory provided one does not consider higher derivative terms ${ }^{11}$. Thus to get a quantum (or at least, quantum-corrected effective) theory which obeys some notion of covariance and is yet different from GR one needs to have a some deformation in the hypersurface deformation algebra. Since we assumed that the diffeomorphism constraint remains unmodified, our only deformation could appear in the brackets between the Hamiltonian constraint and consequently, we $d o$ end up with a non-classical version of space-time although our classical hypersurfaces retain their classical form. Hence, this is an additional argument, which is consistent with our findings, that a deformed notion of covariance is required also from the point of the uniqueness theorem due to Hojman et al.

It must be appreciated that modifying the constraints and yet attaining a closed algebra can turn out to be an extremely ambitious task to accomplish. This has been demonstrated recently in the works of [42], where the authors wished to generalize the constraints by including non-canonical kinetic terms. Such higher derivative kinetic terms were shown to be severely restrictive since the resulting algebra was shown to suffer from anomalies. The underlying reason for this goes back to the idea that modifying gravity is rather more difficult than what it seems like at first. Let us look at the problem from another angle. In the Lagrangian formulation one might say that the quantum-corrected effective (gravitational) action takes the form

$$
S[g]=\frac{1}{16 \pi G} \int \mathrm{d}^{4} x \sqrt{-g}[R+\cdots]
$$

where the dots signify corrections to the Einstein-Hilbert action coming from loop corrections. Any such term must itself be covariant in the sense that it is built out of curvature invariants and have their Lorentz indices contracted in a proper way. But these corrections, local or nonlocal, still leads to the same Dirac algebra since the theory remains covariant after including them. However, there can be one further correction possible in the above context - one might find a redefinition of the measure ' $\mathrm{d}^{4} x \sqrt{-g}$. This would mean that the notion of covariance in the theory is now changed. However, such changes must also follow certain rules, which in the canonical theory is implemented by requiring that there are no

\footnotetext{
${ }^{11}$ Indeed, it is known that higher curvature theories also share the same classical hypersurface deformation algebra [41.
} 
anomalies in the theory. This is precisely what one finds in LQG: a new deformed notion of covariance which renders the invariant line-element non-existent in the deep quantum regime. However, if one finds that quantum corrections lead to the Dirac algebra giving rise to anomalies, as is the case for [42], then such corrections must be discarded, at least in their present form. In this context, one must remember that our holonomy-corrections are well-defined local functions for extrinsic curvature compoenents, and not like higher time-derivative kinetic terms as used in [42. Hence, we are easily able to avoid their no-go theorems.

\subsection{What does quantum space-time consist of?}

The most provoking question raised by our essay is regarding the explicit nature of the fundamental space-time. We have shown that they cannot be described by conventional classical Riemannian structures once holonomy modifications from LQG are taken into account. Although we start from a metric, and then define triads in the classical theory, it turns out that once these corrections are included, one cannot reverse the process to go back to the metric picture. In lower curvature regimes one has access to effective Riemannian space-times, and one gets back the usual constructions. However, in the deep quantum regime, one can locally identify an Euclidean or a Lorentzian patch but there is no global metric structure which can represent the full space-time.

We know what quantum space-time is not; it is not the usual Riemannian manifolds we have grown to get accustomed with. However, what remains to be investigated is the explicit nature of the 'atoms' of space-time. Can there perhaps be a non-commutative, or even fractal, geometry, replacing the Riemannian one, at the elemental quantum level? One way to answer this question might be to assume that the space-time has a, say, non-commutative character. Then one can evaluate the same Dirac algebra for such a mathematical construction. If the resulting deformation has a similar nature to that of LQG, it would imply that LQG hints towards such a more fundamental geometry. In that case, we would have to come up with a suitable coarse-graining for such a quantum theory which would be consistent with our understanding of smooth, continuous Riemannian manifolds of classical gravity. There are reasons for suspecting such a relationship between LQG and exotic geometries coming from other considerations. If the hypersurface deformation algebra is deformed due to holonomy corrections as described above, it can be shown that the flat Minkowski limit of it gives rise to a non-commutative $\kappa$-Poincaré space-time [27, 43]. To make this relationship more robust, one needs to establish this correspondence beyond the flat limit.

It is currently a matter of debate as to how should one think about signature-change arising from LQG. Firstly, it needs to be emphasized that the main mathematical concept arising out of holonomy corrections is the idea of 'deformed covariance', with signaturechange being the main physical effect of that. It seems that LQG provides a physical 'Wick-rotation' to be realized, as was envisioned in theories such as the Hartle-Hawking 
(HH) proposal [44] ${ }^{12}$, However, there are also crucial differences in the signature-change emerging in LQG and the $\mathrm{HH}$ proposal. The quantum wave-function typically obeys a difference equation in LQG, as opposed to a more-familiar differential equation in $\mathrm{HH}$, and is closer spirit to the proposal by Vilenkin [45]. There is another way to approach the problem which relies on noticing the fractal-dimensional nature of the theory. One can estimate the effective (spectral) dimension of space-time in quantum gravity theories using methods involving diffusion of particles on a given space-time. These have led to the evaluation of the UV dimension of theories such as Causal Dynamical Triangulations and Asymptotic Safety. Including holonomy modification to the constraints in LQG, one can calculate such UV dimension for the theory. But there is something unique in this case due to signature-change. One gets a 'physical' cut-off for momentum when evaluating integrals due to the fact that the deformation function changes sign. When this function goes to zero, on the 'signature-changing hypersurface', it gives an equation for a upper (UV) limit of the physical momentum which can be achieved in the Lorentzian phase. What we obtain after that is a fuzzy Euclidean space. Preliminary calculations have shown that the spectral dimension for LQG, including holonomy corrections, goes towards the magic value of ' 2 '. However, it does not flow smoothly from 2 to 4 in a linear manner, but rather exhibits a rich multi-fractional character. Simply put, it means that, at different energies, the dimension of space-time is different in the theory.

But why do we keep referring to the Euclidean phase as fuzzy? Well, for once it is not known what is on the other side of this phase. There might have been a Lorentzian phase before it, as is indicated by the cyclic, bouncing models of minisuperspace quantizations of the background in LQG. However, it might just be that there was simply a quantum Euclidean phase till our usual Lorentzian phase popped up. Remember, in the Euclidean patch, one cannot ask temporal questions and hence it makes no sense to ask how 'long' did it last for. There is another reason to suspect that this phase is not just a simple classical, Euclidean geometry. Although it can be seen from the deformed Dirac algebra that the signature of the background is the same as that for Euclidean geometry, the constraints themselves are not the ones arising from Euclidean GR. In some sense, this is what is to be expected after all. The usual Riemannian structure is replaced by a quantum space-time lacking classical analogues. We can, 'effectively', still understand some of its features through the deformed algebra structure. However, if space-time is truly composed of discrete packets which obey some exotic, non-commutative or fractal behaviour, it is only natural that the resulting system cannot be fully understood by classical notions. This forms another reason for the epithet 'fuzzy' to be assigned to this region - in the anticipation that the quantum geometry may turn out to be non-commutative, and perhaps some version of the fuzzy sphere. However, what is now well-understood for certain is that there is a 'fuzzy' quantum region if we go to very high energies, which is non-singular and is certainly not described by well-understood Riemannian structures.

\footnotetext{
${ }^{12}$ Let us illustrate this point with another well-understood analogy. Many interesting physical consequences are known for coupling between long and short-wavelength cosmological modes in the early universe. In a similar vein, it has been shown that LQG can lead to such couplings spelling out a physical mechanism for it.
} 
Finally, once there is no more an invariant line-element, it is not possible to define an action without the usual invariant measure for the integral. This makes working in the Lagrangian picture impossible. However, the Hamiltonian picture remains well-defined with rigorous definitions of gauge-invariant quantities which might be evaluated to calculate observables. It seems that the Hamiltonian formulation, which is on the same footing as the Lagrangian one in the classical setting, is now somehow more preferred in the quantum theory. This is a surprising twist of turns in LQG which would be interesting to explore further. This is, however, what was once prophesied by Dirac ${ }^{13}$ and it seems to be arising from a quantum theory of gravity.

\section{Summary}

We have shown that non-classical structures can arise in quantum gravitational theories with explicit examples of symmetry-reduced models in LQG. As outlined in this essay, preliminary results suggest that emergent 'time' is a concept central to LQG and therefore, this has serious consequences for physical systems such as the big-bang cosmology or black hole singularities. Non-singular signature-change replaces classical singularity in a way much more intricate than what was previously predicted in strict minisuperspace quantizations, through a quantum 'bounce'. A fuzzy Euclidean region inside the core of black holes has new possibilities for the resolution of the information loss paradox, especially in its partial similarity to the black hole complementarity paradigm [36], whereas replacing the big-bang singularity with an 'asymptotically silent' phase is reminiscent of the Hartle-Hawking wavefunction and similar results from Causal Dynamical Triangulations [47]. Thus non-singular bounce from LQG seems to find common ground with other quantum gravity theories, which has been a very tough proposition for these different approaches traditionally. The real question, however, remains whether such exotic constructs are realized in nature. To further probe this question, one first needs to find a full theory of canonical quantum gravity which retains this feature of 'emergent time', going beyond simplified toy models.

\section{References}

[1] John C. Collins, Renormalization, volume 26 of Cambridge Monographs on Mathematical Physics, Cambridge University Press, Cambridge, 1986

[2] Michael B. Green, John H. Schwarz, and Edward Witten, Superstring Theory25th Anniversary Edition, Cambridge University Press, 2012

[3] J. Polchinski, String theory. Vol. 1: An introduction to the bosonic string, Cambridge University Press, 2007

\footnotetext{
${ }^{13}$ Dirac: "It would be permissible to look upon the Hamiltonian form as the fundamental one, and there would then be no fundamental four-dimensional symmetry in the theory" 46]
} 
[4] J. Polchinski, String theory. Vol. 2: Superstring theory and beyond, Cambridge University Press, 2007

[5] K. Becker, M. Becker, and J. H. Schwarz, String theory and M-theory: A modern introduction, Cambridge University Press, 2006

[6] Thomas Thiemann, Modern canonical quantum general relativity, Cambridge University Press, 2008, [gr-qc/0110034]

[7] Abhay Ashtekar and Jerzy Lewandowski, Background independent quantum gravity: A Status report, Class. Quant. Grav. 21 (2004) R53, [gr-qc/0404018]

[8] Leonardo Rastelli, String field theory, In Prepared for the Encyclopedia of Mathematical Physics, Elsevier (2006), 2005, [hep-th/0509129]

[9] Muxin Han and Ling-Yan Hung, Loop Quantum Gravity, Exact Holographic Mapping, and Holographic Entanglement Entropy (2016), [arXiv:1610.02134]

[10] S. W. Hawking and G. F. R. Ellis, The Large Scale Structure of Space-Time, Cambridge Monographs on Mathematical Physics. Cambridge University Press, 2011

[11] Martin Reuter and Frank Saueressig, Quantum Einstein Gravity, New J. Phys. 14 (2012) 055022, [arXiv:1202.2274]

[12] Jennifer Lin, Matilde Marcolli, Hirosi Ooguri, and Bogdan Stoica, Locality of Gravitational Systems from Entanglement of Conformal Field Theories, Phys. Rev. Lett. 114 (2015) 221601, [arXiv:1412.1879]

[13] Erik P. Verlinde, On the Origin of Gravity and the Laws of Newton, JHEP 04 (2011) 029, [arXiv:1001.0785]

[14] Martin Bojowald, Absence of singularity in loop quantum cosmology, Phys. Rev. Lett. 86 (2001) 5227-5230, [gr-qc/0102069]

[15] Abhay Ashtekar, Singularity Resolution in Loop Quantum Cosmology: A Brief Overview, J. Phys. Conf. Ser. 189 (2009) 012003, [arXiv:0812.4703]

[16] Abhay Ashtekar, Martin Bojowald, and Jerzy Lewandowski, Mathematical structure of loop quantum cosmology, Adv. Theor. Math. Phys. 7 (2003) 233-268, [grqc/0304074]

[17] Rodolfo Gambini and Jorge Pullin, Loop quantization of the Schwarzschild black hole, Phys. Rev. Lett. 110 (2013) 211301, [arXiv:1302.5265]

[18] Martin Bojowald, Canonical Gravity and ApplicationsCosmology, Black Holes, and Quantum Gravity, Cambridge University Press, 2010 
[19] Abhay Ashtekar and Parampreet Singh, Loop Quantum Cosmology: A Status Report, Class. Quant. Grav. 28 (2011) 213001, [arXiv:1108.0893]

[20] Martin Bojowald, Mathematical structure of loop quantum cosmology: Homogeneous models, SIGMA 9 (2013) 082, [arXiv:1206.6088]

[21] Abhay Ashtekar, Alejandro Corichi, and Parampreet Singh, Robustness of key features of loop quantum cosmology, Phys. Rev. D77 (2008) 024046, [arXiv:0710.3565]

[22] Daniel Martin de Blas, Javier Olmedo, and Tomasz Pawlowski, Loop quantization of the Gowdy model with local rotational symmetry (2015), [arXiv:1509.09197]

[23] Alejandro Corichi, Javier Olmedo, and Saeed Rastgoo, Vacuum CGHS in loop quantum gravity and singularity resolution, Phys. Rev. D94 (2016) 084050, [arXiv:1608.06246]

[24] Martin Bojowald, Quantum Cosmology: Effective Theory, Class. Quant. Grav. 29 (2012) 213001, [arXiv:1209.3403]

[25] Martin Bojowald and Suddhasattwa Brahma, Covariance in models of loop quantum gravity: Gowdy systems, Phys. Rev. D92 (2015) 065002, [arXiv:1507.00679]

[26] Martin Bojowald, Suddhasattwa Brahma, and Juan D. Reyes, Covariance in models of loop quantum gravity: Spherical symmetry, Phys. Rev. D92 (2015) 045043, [arXiv:1507.00329]

[27] Suddhasattwa Brahma, Michele Ronco, Giovanni Amelino-Camelia, and Antonino Marciano, Linking loop quantum gravity quantization ambiguities with phenomenology, Phys. Rev. D95 (2017) 044005, [1610.07865]

[28] Martin Bojowald and Rafal Swiderski, Spherically symmetric quantum geometry: Hamiltonian constraint, Class. Quant. Grav. 23 (2006) 2129-2154, [gr-qc/0511108]

[29] Martin Bojowald, George M. Paily, and Juan D. Reyes, Discreteness corrections and higher spatial derivatives in effective canonical quantum gravity, Phys. Rev. D90 (2014) 025025, [arXiv:1402.5130]

[30] Suddhasattwa Brahma, Spherically symmetric canonical quantum gravity, Phys. Rev. D91 (2015) 124003, [arXiv:1411.3661]

[31] Alok Laddha and Madhavan Varadarajan, The Diffeomorphism Constraint Operator in Loop Quantum Gravity, Class. Quant. Grav. 28 (2011) 195010, [arXiv:1105.0636]

[32] Martin Bojowald and George M. Paily, Deformed General Relativity, Phys. Rev. D87 (2013) 044044, [arXiv:1212.4773]

[33] Martin Bojowald and Suddhasattwa Brahma, Effective constraint algebras with structure functions, J. Phys. A49 (2016) 125301, [arXiv:1407.4444] 
[34] Martin Bojowald and Jakub Mielczarek, Some implications of signature-change in cosmological models of loop quantum gravity, JCAP 1508 (2015) 052, [arXiv:1503.09154]

[35] Thomas Cailleteau, Jakub Mielczarek, Aurelien Barrau, and Julien Grain, Anomalyfree scalar perturbations with holonomy corrections in loop quantum cosmology, Class. Quant. Grav. 29 (2012) 095010, [arXiv:1111.3535]

[36] Martin Bojowald and Suddhasattwa Brahma, Signature change in 2-dimensional black-hole models of loop quantum gravity (2016), [arXiv:1610.08850]

[37] Martin Bojowald and Suddhasattwa Brahma, Signature change in loop quantum gravity: General midisuperspace models and dilaton gravity (2016), [arXiv:1610.08840]

[38] Martin Bojowald, Suddhasattwa Brahma, Umut Buyukcam, and Fabio D'Ambrosio, Hypersurface-deformation algebroids and effective space-time models (2016), [arXiv:1610.08355]

[39] S. A. Hojman, K. Kuchar, and C. Teitelboim, Geometrodynamics Regained, Annals Phys. 96 (1976) 88-135

[40] K. Kuchar, Geometrodynamics regained - a lagrangian approach, J. Math. Phys. 15 (1974) 708-715

[41] Nathalie Deruelle, Misao Sasaki, Yuuiti Sendouda, and Daisuke Yamauchi, Hamiltonian formulation of $\mathrm{f}($ Riemann) theories of gravity, Prog. Theor. Phys. 123 (2010) 169-185, [arXiv:0908.0679]

[42] Henrique Gomes and Vasudev Shyam, Extending the rigidity of general relativity, $J$. Math. Phys. 57 (2016) 112503, [1608.08236]

[43] Jakub Mielczarek and Tomasz Trzeniewski, Spectral dimension with deformed spacetime signature (2016), [1612.03894]

[44] J. B. Hartle and S. W. Hawking, Wave Function of the Universe, Phys. Rev. D28 (1983) 2960-2975

[45] A. Vilenkin, Quantum Creation of Universes, Phys. Rev. D30 (1984) 509-511

[46] P.A.M Dirac, Generalized Hamiltonian dynamics and the theory of gravitation in Hamiltonian form, Proc. R. Soc. Lond. A (1958) 333

[47] Jakub Mielczarek, Asymptotic silence in loop quantum cosmology (2012), [arXiv:1212.3527], AIP Conf. Proc.1514,81(2012) 